\title{
Autogenous Tooth Bone Graft Block For Socket Preservation: A One-Stage Technique
}

\author{
Young Kyun Kim ${ }^{1,2}$, Kimberly Ray R Fajardo ${ }^{3}$, Alfred Joseph O Valera ${ }^{4}$ and In-Woong Um ${ }^{5^{*}}$ \\ ${ }^{1}$ Department of Oral and Maxillofacial Surgery, Section of Dentistry, Seoul National University Bundang Hospital, Seongnam, Korea \\ ${ }^{2}$ Department of Dentistry \& Dental Research Institute, School of Dentistry, Seoul National University, Seoul, Korea \\ ${ }^{3}$ Prosthodontics and Undergraduate Research Section, University of the East College of Dentistry, Manila, Philippine \\ ${ }^{4}$ Surgery Department, University of the East-College of Dentistry, Manila, Philippine, Philippine College of Oral and Maxillofacial Surgery, Manila, Philippine \\ ${ }^{5} R \& D$ Institute, Korea Tooth Bank, Seoul, Korea
}

*Corresponding author: In-Woong Um, Director, R\&D Institute, Korea Tooth Bank, Seoul-In Dental Clinic, 622, Eonju-ro, Gangnam-gu, Seoul, 135-832, Korea Republic Of, Tel: +8225482055; Fax: + 8225482228; E-mail: h-bmp@hanmail.net

Received date: Feburary 08, 2017; Accepted date: Feburary 20, 2017; Published date: February 28, 2017

Copyright: (c) $2017 \mathrm{Kim}$ YK, et al. This is an open-access article distributed under the terms of the Creative Commons Attribution License, which permits unrestricted use, distribution, and reproduction in any medium, provided the original author and source are credited.

\begin{abstract}
To replace the autogenous bone block graft in socket preservation technique that has disadvantages despite gold standard such as second surgery, more healing period and cost, the author introduces the surgical technique and five-year clinical outcome of using preformed autogenous tooth bone graft block (ABTB) which was fabricated from the patient's own extracted tooth, with simultaneous implant placement. Evaluations of the five-year clinical outcome were also performed with cone beam computed tomography (CBCT).

ABTB is root dentin block composed of Type I collagen matrix with several osteoinductive non-collagenous proteins such as bone morphogenetic proteins (BMP) and dentin matrix proteins. Geometrically, alveolar bone morphology was maintained by ABTB as well as it has micropores (dentinal tubules) of 3-5 $\mu \mathrm{m}$ diameter and macropores of 0.2-0.3 mm diameter for enhancing osteoinductive and osteoconductive functions.

Five year follow up with CBCT showed that alveolar bone volume and shape were maintained successfully which was repaired and restored by $\mathrm{ABTB}$. This might become the effective technique for socket preservations given the well controlled long term follow up studies.
\end{abstract}

Keywords: Autogenous tooth bone graft block (ABTB); Demineralized dentin matrix (DDM); Cortico-cancellous bone

\section{Introduction}

Many studies have reported an approximately 50\% reduction in alveolar bone both the horizontal and vertical directions over 12 months with more than two-thirds of the reduction occurring in the first three months after extraction [1-3]. In implant dentistry, the buccal alveolar ridge width should be at least $2 \mathrm{~mm}$ if the alveolar bone level on the facial aspect is to be maintained. If this minimal requirement is not met, an augmentation ridge procedure (before or at implant placement) should be performed to achieve this minimal dimension [4-6].

The augmentation of the extraction sites with graft materials tends to reduce this bone loss, most likely via the maintenance of physical stimulation of the surrounding bone. In addition, immediate implant placement has advantages, including the prevention of alveolar bone resorption. A simultaneous immediate implant placement and ridge preservation procedure have been proposed to prevent serious bone resorption $[6,7]$.

Autogenous block bone grafts are applied for alveolar ridge reconstruction in wide three dimensional bone defects in edentulous spaces. Taken from intraoral or extraoral donor sites, autogenous block bone grafts might be free cancellous or corticocancellous. Autogenous grafts contain a variety of living cells and growth factors that have osteoinductive, osteoconductive and osteogenic effects [8-10]. The fact that autogenous block bone grafts have osteoinductive, osteoconductive and volume enhancement properties makes them ideal for the reconstruction of three-dimensional alveolar bone defects. However, the increased treatment period by the second surgery and healing period for harvesting block bone and cost are taken into consideration when the implant plecement is planned.

An autogenous tooth bone graft block (ABTB), which is fabricated from root dentin as a substitute for the autogenous bone block, is a biomimetic of cortical bone that exhibits slow creeping substitution properties with 3-5 $\mu \mathrm{m}$ innate micropores (dentinal tubules) and $0.2-0.3 \mathrm{~mm}$ macropores (Figure 1). Not only the macropores provide the space for vascular invasion from the host tissues but also the micropores (innate dentinal tubules) enable the Type I collagen matrix to exchange proteins between the dentin matrix block and host tissues.

ABTB has osteoconductive, osteoinductive, and volume maintaining capacities mainly due to its 10 to $30 \%$ mineral contents after demineralization. In addition, the geometrical similarity of ABTB to the alveolar bone has major advantages required in threedimensional scaffolds because ABTB outcontours the alveolar bone before extraction, which is needed for the site of future implants [11-13]. 
Page 2 of 5

Indications for the ABTB include socket preservation and vertical augmentation in cases that the socket wall is expected to be resorbed or has already been destroyed due to a range of pathological causes. An advantage of the ABTB is that its geometry adapts well to the graft site because the ABTB is pre-positioned at the site of the implant and possesses the same components of mineral, type I collagens as alveolar bone $[12,14]$.

The author suggests a strategy of an immediate implant along with ABTB in the same time for socket preservation. This article gives the definition to a surgical technique of repair and restoring alveolar ridges using $\mathrm{ABTB}$ at the time of simultaneous implant placement.

\section{Materials and Methods}

\section{Fabrication of ABTB}

The extracted wisdom tooth was immersed in $75 \%$ alcohol. After removing the soft tissues and calculus attached the tooth, crowns were severed at the cementoenamel junctions. Only root dentin part was processed for the ABTB fabrication (European Patent No. 2462899) for its intended use as described in the previous report [12]. Additional holes sized in $0.2 \mathrm{~mm}$ were made at the surface of the canal area to create macropores for promoting vascular invasion and bone formation. The ABTB went through the same fabrication process with the powder form, but the only difference was not being crushed into pieces so that ABTB maintains the original tooth root shape (Figure 1).

The ABTB, after rehydration, can be molded and trimmed with a surgical knife because of the resilient nature due to demineralization (Figure 1). As a result, the buccal root of the second premolar can be set back to its original position, resulting in socket preservation and vertical augmentation.

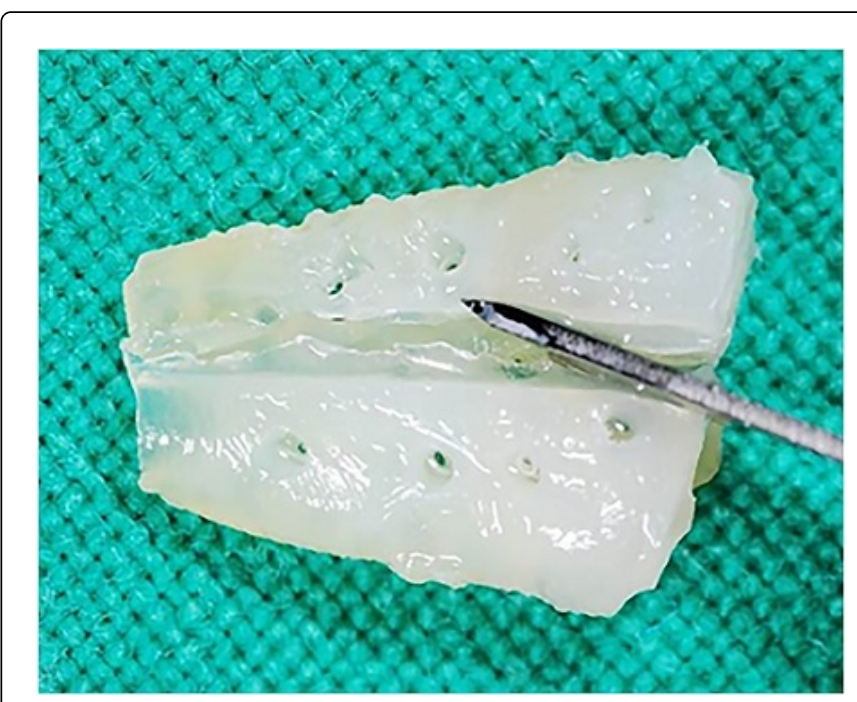

Figure 1: $A B T B$ fabricated from extracted second premolar. Trimming ABTB with a surgical knife to insert between the implant and remained buccal cortical bone. The buccal root below the cemento-enamel junction was used and a 1-2 mm apex was removed for geometrical adaptation.

\section{Case 1. Socket preservation, vertical augmentation, and repairing dehiscence \#15}

A 45-year-old woman presented with a complicated apical lesion with an abscess, pain, and mobility of the upper right second premolar. The patient had a history of several apicoectomies over the past 10 years. Cone Beam Computed Tomography (CBCT) revealed severe apical bone resorption with radiolucency and remained buccal cortical plate less than a $2-\mathrm{mm}$ thickness, that was expected to be resorbed after extraction (Figure 2A).

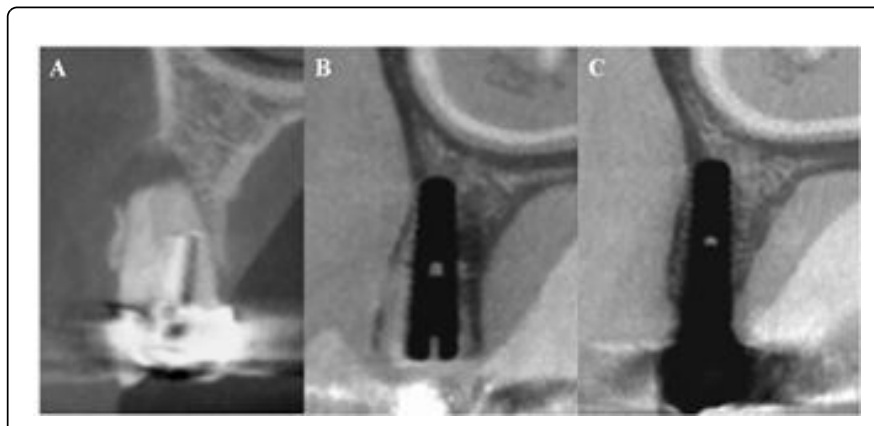

Figure 2: Cone Beam Computed Tomograph of Case 1. (A) Preoperative CBCT showed severe apical bone resorption with radiolucency and buccal cortical plate less than 2-mm thickness, that was expected to be resorbed after extraction. (B) CBCT immediate after surgery showed that $\mathrm{ABTB}$ maintained the buccal aspect of the remaining cortical plate with implant. (C) Five years later, the buccal aspect of alveolar bone around the implant had maintained its volume and shape. Buccal cortical bone was supportd by well developed cancellous bone.

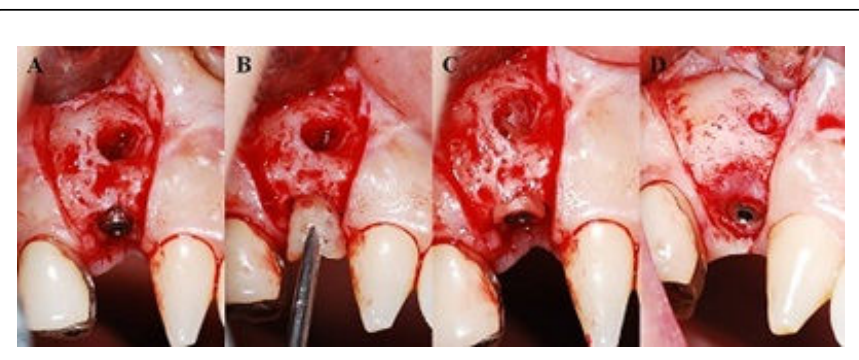

Figure 3: Clinical Pictures of Case 1. (A) Apical dehiscence and vertical height deficiency around the buccal crestal area was observed after implant $\left(\varnothing 3.8 \times 12 \mathrm{~mm}\right.$, Dentis ${ }^{\oplus}$, Seoul, Korea $)$ placement in accordance with the palatal bone level and adjacent alveolar bone margin. (B) The buccal root of the ABTB was dissected and molded to fit into second premolar and was set back to position where it was betwen the buccal cortical plate and implant fixture. (C) ABTB inserted between the buccal cortical plate and implant not only repaired the apical dehiscence, but also augmented 2-mm high vertical deficiency. (D) Four months later, when the flap was reopened for the prosthetic procedure, we could find the ABTB repairing the apical dehiscence and augmenting vertical deficiency.

Seven to ten days after extraction, with the ABTB fabricated from the second premolar removed, socket preservation was performed with a repair of the apical dehiscence and vertical augmentation of the 
buccal crestal bone. CBCT immediately after surgery showed that ABTB maintained the buccal aspect of the remaining cortical plate with implant (Figure 2B).

Five years later, the buccal aspect of alveolar bone around the implant had maintained its volume and shape. Buccal cortical bone is supportd by well developed cancellous bone. Also the quality of bone measured in pixels had increased remarkably from an average 854 to 2214 (Figure 2C).

A buccal height deficiency was observed after placing the implant $\left(3.8 \times 12 \mathrm{~mm}\right.$, Dentis ${ }^{\otimes}$, Seoul, Korea) in accordance with the palatal bone and adjacent alveolar bone margin (Figure 3A). The ABTB, which was fabricated from the extracted second premolar, was molded and trimmed with a surgical knife (Figure 1) for insertion between the implant and remaining thin buccal cortical plate (Figure 3B). As a result, the buccal root of the second premolar was set back to position where it was, resulting in vertical augmentation and repairing the labial dehiscence (Figure 3C). The remodeling of the ABTB was observed four months later (Figure 3D).

\section{Case 2. Socket preservation \#14}

A 58-year-old woman presented with complicated periodontosis, including severe pain and mobility on the upper right first premolar. CBCT revealed a swollen periodontal ligament, moderate alveolar bone resorption, and apical radiolucency with a thin buccal cortical plate less than $1 \mathrm{~mm}$.

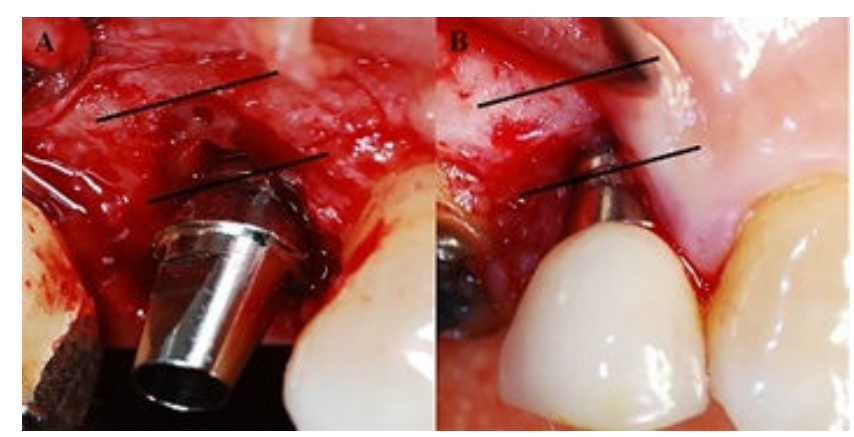

Figure 4: Clinical Pictures of Case 2. (A) ABTB was inserted between the thin buccal cortical plate and implant. Provisional prosthetic abutment was then placed. The color change of the ABTB is mainly due to soaking with the patient's own blood into the macropores and collagenous dentin matrix. (B) Five years later, the $\mathrm{ABTB}$ inserted has changed into mature bone with its volume and quality maintained.

The implant was placed after extracting the first premolar, and then the ABTB was inserted between the buccal cortical plate and implant fixture as in case 1. Provisional prosthetic abutment was placed and the socket was closed to be watertight around the abutment neck area. The ABTB was fully soaked with patient's own blood via its collagenous nature and macropores (Figure 4A). Five years later, the flap on the first premolar was opened with the patient's consent by way of the surgical treatment to the adjacent second premolar. The ABTB inserted has changed into mature bone with its volume and quality maintained (Figure 4B).
CBCT taken immediately after the graft showed the ABTB with macropores between thin buccal cortical plate less than $1 \mathrm{~mm}$ thickness and the implant (Figure 5A). All healing processes were uneventful, the implant integrated well, and the ABTB was well incorporated with the buccal wall through continuous remodeling one year after the graft (Figure 5B). After five years, the bone around the implant showed a cortico-cancellous complex with more than $2 \mathrm{~mm}$ thickness. The quality of bone measured in pixels had increased from an average of 1113 to 1784 (Figure 5C).

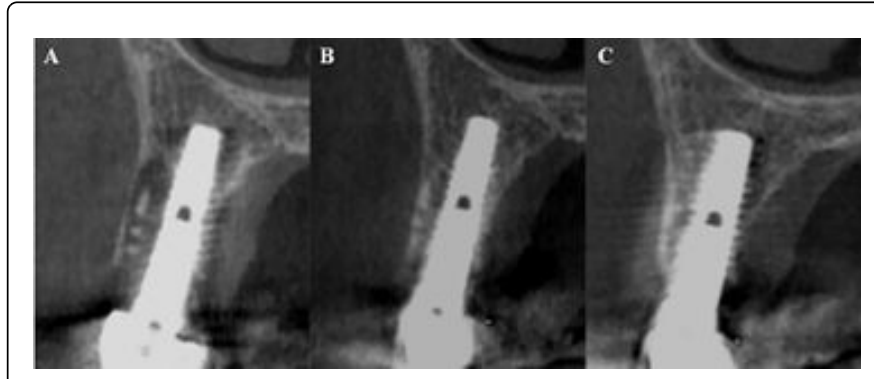

Figure 5: Cone Beam Computed Tomograph of Case 2. (A) CBCT taken immediate after the graft shows the ABTB with macropores between the implant and thin buccal cortical bone. (B) One year after the graft, СBCT showed that the implant was well integrated and the ABTB was well incorporated with the buccal cortical bone under continuous remodeling, which has maintained the buccal alveolar bone volume. (C) After five years, the bone around the implant showed a sound cortico-cancellous complex.

\section{Discussion}

The treatment performed with intra-oral autogenous block graft is one of the best methods to increase the insufficient bone volume on the region where implant is to be placed. Thin residual bone remaining in the buccal region after tooth removal is quickly resorbed. Generally, a reduction in alveolar bone volume appears to be caused by progressive resorption, with a loss of $0.34-7.7 \mathrm{~mm}$ of ridge width and 0.2-3.25 $\mathrm{mm}$ of vertical height during the 6-12 months following extraction. But socket preservation is successfully performed due to the reconstruction of socket with autologous bone graft $[15,16]$. With the bone reconstruction in the same session with the implant placement, possible bone resorption can be prevented [17].

The reconstruction of alveolar defects with autogenous block graft is considered to be the gold standard. However, this treatment requires two different surgical procedures and takes much time [18]. In this case report, the ABTB was used to replace autogenous bone block graft not only for reducing the disadvantages of autogenous bone block but also for enhancing the predictability of socket preservation.

Generally, the bone substitutes should be estimated whether it has the properties of autologous bone that promote the healing process or how well do they match autologous bone grafts. Regarding the properties of healing, ABTB has almost same composition with autologous bone such as Type I collagen, minerals and several proteins $[14,19,20]$. Structurally, ABTB has 0.2-0.3 mm diamter macropores to enhance vascular invasion from the host tissues and 3-5 $\mu \mathrm{m}$ diameter micropores for exchanging proteins between host tissues and dentin matrix. 
These are the reasons why ABTB showed the osteoinductive and osteoconductive properties. It also has adequate geometrical similarity so that it fits into the socket, as it is positioned and structured according to the existing alveolar bone. Moreover, easy handing and trimming on the buccal contour makes ABTB more feasible [12]. Similarly, retaining a root for alveolar ridge preservation has been tested in several clinical studies.

Kim et al. reported that the osteoinductive and osteoconductive functions of ABTB were verified by histological examination $[19,20-22]$. Kim et al. also reported in the long term follow up study of ABTB that CBA, which were $41.78 \%$ in the mandible and $28.28 \%$ in the maxilla, appear to be closely related to decreases in $\mathrm{BH}$ because his phenomenon was mainly caused by a reduction in $\mathrm{BH}(17.03 \%$ in the maxilla and $40.8 \%$ in the mandible) rather than ARW $(21.18 \%$ in the maxilla and $24.61 \%$ in the mandible) [23].

These findings from long-term follow-up are also promising relative to other results, which indicated that the mean resorption volumes for 14 patients with 32 iliac and chin grafts at one year after implant positioning were $35-51 \%$. In contrast, mean volume reductions of $16.2 \%$ at 6 months (15 patients) and $19.2 \%$ at 12 months (five patients) were observed in other studies [24]. Verhoeven et al. reported a $36 \%$ mean resorption rate for the graft, with resorption mainly occurring during the first year after surgery [25].

Even though our case reports showed more resorption of buccal area $54.1 \%$ (Case 1) and $54.6 \%$ (Case 2) than the long term follow up report (average 28.28\%), not only the volume and shape of corticocancellous bone were maintained but also the quality of alveolar bone were continuously increased from an average 854 to 2214 (case 1) and an average of 1113 to 1784 (case 2) in both the cases [23].

Despite the discussed advantages of ABTB grafts, they also have a few limitations. Tooth extraction is necessary to create an ABTB graft. The number of teeth available for extraction is limited, resulting in an unpredictable amount of bone graft material that can be obtained [26]. In this case report, the application of ABTB combined with immediate implant placement maintained the bone volume and shape for more than 5 years. These two cases fulfilled the success criteria of implant dentistry in terms of the universally accepted peri-implant remodeling $[27,28]$. Further studies of well-designed, high quality, randomized clinical trials are required to validate the efficacy of this technique.

\section{Acknowledgement}

This study was supported by the Korea Health Industry Institute (KHIDI) grant that is funded by the ministry of Health \& Welfare, Republic of Korea (Grant number: HI15C3136).

\section{References}

1. Dimova C (2014) Socket Preservation Procedure after Tooth Extraction. Key Engineering Materials 587: 325-330.

2. Tan WL, Wong TL, Wong MC, Lang NP (2012) A systematic review of post-extractional alveolar hard and soft tissue dimensional changes in humans. Clinical Oral Implants Research 23: 1-21.

3. Brkovic BM, Prasad HS, Konandreas G, Milan R, Antunovic D, et al. (2008) Simple preservation of a maxillary extraction socket using betatricalcium phosphate with type I collagen: preliminary clinical and histomorphometric observations. J Can Dent Assoc 74: 523-528.

4. Huynh-Ba G, Pjetursson BE, Sanz M, Cecchinato D, Ferrus J, et al. (2010) Analysis of the socket bone wall dimensions in the upper maxilla in relation to immediate implant placement. Clin Oral Impl Res 21: 37-42.
5. Belser U, Martin W, Jung R, Hammerle C, Schmid B, et al. (2007) ITI Treatment Guide, Volume 1: Implant Therapy in the Esthetic Zone. Single-tooth Replacements. Quintessence Publishing, Berlin.

6. Becker W (2005) Immediate implant placement: diagnosis, treatment planning and treatment steps/or successful outcomes. J Calif Dent Assoc 33: 303-310.

7. Iasella JM, Greenwell H, Miller RL, Hill M, Drisko C, et al. (2003) Ridge preservation with freeze-dried bone allograft and a collagen membrane compared to extraction alone for implant site development: a clinical and histologic study in humans. J Per 74: 990-999.

8. Misch CE, Dietsh F (1993) Bone-grafting materials in implant dentistry. Implant Dent 2: 158-167.

9. Marx RE (1994) Clinical application of bone biology to mandibular and maxillary reconstruction. Clin Plast Surg 21: 377-392.

10. Cordaro L, Amade DS, Cordaro M (2002) Clinical results of alveolar ridge augmentation with mandibular block bone grafts in partially edentulous patients prior to implant placement. Clin Oral Implants Res 13:103-111.

11. Kim YK, Kim SG, Byeon JH, Lee HJ, Um IU, et al. (2010) Development of a novel bone grafting material using autogenous teeth. Oral Surgery, Oral Medicine, Oral Pathology, Oral Radiology, and Endodontology 109: 496-503.

12. Kim YK, Um IW, Murata M (2014) Tooth bank system for bone regeneration - safety report. Journal of Hard Tissue Biology 23: 371-376.

13. Murata M (2003) Autogenous demineralized dentin matrix for maxillary sinus augmentation in humans: the first clinical report. Journal of Dental Research 82: B 243.

14. Murata M (2012) Collagen biology for bone regenerative surgery. J. Korean Assoc Oral Maxillofac Surg 38: 321-325.

15. Schropp L, Kostopoulos L, Wenzel A (2003) Bone healing following immediate versus delayed placement of titanium implants into extraction sockets: a prospective clinical study. Int J Oral Maxillofac Implants 18: 189-199.

16. Schropp L, Wenzel A, Kostopoulos L, Karring T (2003) Bone healing and soft tissue contour changes following single-tooth extraction: a clinical and radiographic 12-month prospective study. Int J Periodontics Restorative Dent 23: 313-323.

17. Barzilay I (1993) Immediate implants: their current status. Int J Prosthodont 6: 169-175.

18. Zouhary KJ (2010) Bone graft harvesting from distant sites: Concepts and techniques. Oral Maxillofac Surg Clin North Am 22: 301-316.

19. Kim YK, Kim SG, Um IW, Kim KW (2013) Bone grafts using autogenous tooth blocks: a case series. Implant Dent 22: 584-589.

20. Lee EG, Lee JY, Kim YK, Um IW, Choi JH (2013) Delayed Implant Placement after Extraction Socket Reconstruction and Ridge Augmentation using Autogenous Tooth Bone Graft Material: Case Reports. Dentistry 3:174.

21. Hürzeler MB, Zuhr O, Schupbach P, Rebele SF, Emmanouilidis N, et al. (2010) The socket-shield technique: a proof-of-principle report. J Clin Periodontol 37: 855-862.

22. Kim YK, Yun PY, Um IW, Lee HJ, Yi YJ, et al. (2014) Alveolar ridge preservation of an extraction socket using autogenous tooth bone graft material for implant site development: prospective case series. J Adv Prosthodont 6: 521-527.

23. Kim YK, Pang KM, Yun PY, Leem DH, Um IW (2017) Long-term followup of autogenous tooth bone graft blocks with dental implants. Clinical Case Reports 5: 108-118.

24. Smolka W, Eggensperger N, Carollo V, Ozdoba C, Iizuka T (2006) Changes in the volume and density of calvarial split bone grafts after alveolar ridge augmentation. J Clin Oral Implants Res 17: 149-155.

25. Verhoeven JW, Cune MS, Terlou M, Zoon MA, de Putter C (1997) The combined use of endosteal implants and iliac crest onlay grafts in the severely atrophic mandible: a longitudinal study. Int J Oral Maxillofac Surg 26: 351-357. 
Citation: Kim YK, Fajardo KRR, Valera AJO, Um IW (2017) Autogenous Tooth Bone Graft Block For Socket Preservation: A One-Stage Technique. Dentistry 7: 414. doi:10.4172/2161-1122.1000414

Page 5 of 5

26. Jeong KI, Lee J, Um IW (2015) Alveolar Cleft Restoration Using Autogenous Tooth Bone Graft Material for Implant Placement: A Case Report. J Oral Implantol 41:487-490.

27. Albrektsson T, Zarb G, Worthington P, Eriksson AR (1986) The longterm efficacy of currently used dental implants: a review and proposed criteria of success. Int J Oral Maxillofac Implants 1: 11-25.
28. Papaspyridakos P, Chen CJ, Singh M, Weber HP, Gallucci GO (2012) Success criteria in implant dentistry: a systematic review. J Dent Res 91: 242-248. 